\title{
Alteration of Basal Blood Glucose Level and Microbiota Metabolic Parameters in Rats Fed with Brown Rice and Herbal Extracts
}

\author{
Indra Wibowo ${ }^{1, *}$, Intan Taufik ${ }^{1, *}$, Mira Mutiyani ${ }^{2}$, Nuruliawaty Utami ${ }^{1}$, Nurfitri Yulianty ${ }^{1}$, \\ Aam Kamal ${ }^{1}$, Aditya Rinaldy ${ }^{1}$, Roya Suffah Zain ${ }^{1}$, Tjandra Anggraeni ${ }^{1}$ \\ ${ }^{1}$ School of Life Sciences and Technology (SITH), Institut Teknologi Bandung (ITB), Bandung, West Java, Indonesia 40132 \\ ${ }^{2}$ Nutrition Department, Bandung Polytechnic of Health, Ministry of Health, Cimahi, West Java, Indonesia 40514 \\ *Corresponding author: indra@sith.itb.ac.id; i.taufik@sith.itb.ac.id
}

Received January 07, 2019; Revised February 09, 2019; Accepted February 24, 2019

\begin{abstract}
Obesity has been identified as a cause of various diseases. The main solution in dealing with this metabolic syndrome is a healthy lifestyle, with intake of fibrous and herbal extracts. Various studies have shown that gut microbiota involved in many metabolic syndromes. This study aims to determine the effect of brown rice and herbal extracts (Cuminum cyminum and Alpinia galanga) on the physiological profile of rat gut microbiota. The research was done by measuring changes in the physiological profile of the gut microbiota, analyzing metagenome of gut microbiota using Next Generation Sequencing technology, and also observing changes in blood glucose and cholesterol levels for three weeks in normal condition. Changes in the physiological level of significant microbiota were used as reference points at the later stage. The average blood glucose level of rats in the all treatment groups was decreased except for the group treated with the mixture of brown rice and Alpinia galanga. The rats fed with combination of brown rice and herbal extracts showed the highest Average Well Color Development (AWCD) index. Moreover, this treatment group had also the highest degradation activity in almost all types of substrates as observation proceeds. These results suggest that the combination of brown rice with bioactive compounds contained in those extracts provides a healthy gut microbiome. In conclusion, our results showed that the differences in type of food intake can affect the physiological conditions of the hosts' body and metabolic parameters of rat intestinal microbiota.
\end{abstract}

Keywords: herbal extract, Cuminum cyminum, Alpinia galanga, gut microbiota, brown rice, metabolic syndrome

Cite This Article: Indra Wibowo, Intan Taufik, Mira Mutiyani, Nuruliawaty Utami, Nurfitri Yulianty, Aam Kamal, Aditya Rinaldy, Roya Suffah Zain, and Tjandra Anggraeni, "Alteration of Basal Blood Glucose Level and Microbiota Metabolic Parameters in Rats Fed with Brown Rice and Herbal Extracts." Journal of Food and Nutrition Research, vol. 7, no. 2 (2019): 162-170. doi: 10.12691/jfnr-7-2-9.

\section{Introduction}

Gut microbiota has become an important research topic, especially in relation to human health. Obesity and type-2 diabetes in human are already known to be related to changes in the intestinal microbiota [1]. Obesity is also associated with other diseases such as hypertension and heart coronary disease, infertility, and cancer [2]. Nevertheless, direct correlation between gut microbiota and these diseases has not yet fully understood.

Several studies have shown that gut microbiota can undergo changes due to consumption of prebiotic, herbal extract, and fiber [3,4], which in turn promoye improvement of the host immune system [5]. By increasing the consumption of prebiotic, fiber, or herbal extract, it is expected that there will be a change in the gut microbiota, which in turn will improve the body's health. In addition to strengthening immune system, the presence of a health-promoting gut microbiota strongly supports the digestive process and produces various metabolites needed by the body.

In general, Indonesian diet usually consist of high fiber comprising of various types of plant products such as cereals, tubers and others. On the other hand, they also used herbal extracts for prevention and treatment of several diseases, such as extract of Alpinia galanga, Rheum palmatum, Cuminum cyminum, Guazuma ulmifolia, Trigonella fonum graceum, Ganoderma lucidum, and Piper betle as antidiabetic [6]. Through the years, there has been a shift of diet toward high fat foods, and decreased use of herbal extracts to prevent or treat diseases by the community. This change has, in part, resulted in the emergence of metabolic syndromes, including obesity. This is not only a local problem, but also a global issue that even affects early childhood [7].

Previous studies have shown that complementation of probiotics and herbal extracts into diets can lead to changes in the intestinal microbiota, especially the dominant groups [8]. A review illustrates this well, 
specifically related to murine model studies [9]. Although with several differences, the murine model is still considered a useful model that can provide an overview of human system. Resembling several previous studies, in this research, we want to explore the effect of brown rice as high-fiber source complemented with several widely used Indonesian herbal extracts, specifically from $A$. galanga rhizoma and $C$. cyminum seeds. This study begun with a preliminary examination to determine the most significant changes in the physiological profile of the microbial community level to start fiber intake. The change in food intake will certainly have an impact on the gut microbial community.

Various niches in the human body accommodate multitude of microbes known as microbiota. More than 100 trillion microbes forms microbiome, comprising genes, physiological processes and microbial products that provide some benefit to the health of host, including to the immune system homeostasis, food conversion, and protection against harmful pathogens. Nevertheless, different microbiome exist between individuals. Such differences are due to different environment, habits and also diets of the host. It is known that low bacterial diversity play role in the emergence of various disease, including diabetes [10].

Human gut microbiota is composed by bacteria, archaea, protozoans, fungi, etc. The dominant bacterial species are divided into three phyla: Bacteroidetes (e.g. Prevotella), Firmicutes (e.g. Ruminococcus, Clostridium, Eubacteria etc.), and Actinobacteria (e.g. Bifidobacterium). Some bacteria are found in small number, these includes Lactobacilli, Streptococci, and Escherichia coli. Based on metagenomic studies, Bacteroidetes and Firmicutes were found as the dominant populations in the gastrointestinal tract.

As previously mentioned, host's diet play important role in determining the gut microbial composition, whilst host health depend on microbial metabolism [11]. Prebiotics, such as non-digestible carbohydrates or fibers promotes microbial growth, changing its composition, thus provide multiple benefits to the health. Such stimulants promote growth of groups such as bifidobacteria and lactobacilli. Herbal extracts containing fiber, polyphenol, and polysaccharides are known to have anti-obese effects by altering the gut microbiota [12].

In this study, we have observed the physiological profile in microbial community level, and progressed to biodiversity profiling using next generation sequencing (NGS) as it has been found very effective and efficient in dissecting microbial diversity in a community [13], of both culturable and unculturable species [14]. We expect this study would greatly contribute in studying gut microbiota in Indonesia. We also anticipated to show that fiber intake and complementing diets with herbal extracts are important and can provide solution to obesity issues by providing diet type recommendation that considered as healthy and antidiabetic.

Thus, this study aims to study the changes of gut microbiota profile in rats due to administration of brown rice feed combined with herbal extracts of $A$. galanga rhizoma and $C$. cyminum seeds. Our approach is to examine the difference of combined community level physiological profile of the microbiota (Community-Level Physiological Profiling - CLLP) and biodiversity profile based on $16 \mathrm{~S}$ rRNA, before and after feeding of the rats with brown rice and herbal extracts.

\section{Materials and Methods}

\subsection{Brown Rice Feed}

Brown rice used was from Olens ${ }^{\circledR}$ (Bumi Ganesa) certified by Laboratory of the Indonesian Center for Veterinary Research, Ministry of Agriculture (No. LB. 07/558) and Bogor Institute of Agricultures's Center for Biological and Biotechnology Research (No.4 / Analysis / V / 2007 P-IRT Health Service No. 215327301736). Standard rat feed (PC551) was used in mixture with brown rice flour (1:1), applied as pellets.

\subsection{Cuminum cyminum and Alpinia galanga Extraction}

C. cyminum seed and A. galanga rhizome powder were obtained from Local Herbal Store Bandung, West Java, Indonesia. Extracts were made by diluting powder in distillated water [15] that already heated to $100^{\circ} \mathrm{C}$, and strained using filter cloth. Dosages used were $250 \mathrm{mg}$ per $\mathrm{kg}$ body weight for A. galanga extract [15] and $400 \mathrm{mg}$ per kg body weight for C. cyminum [16].

\subsection{Treatment with Brown Rice and Herbal Extract}

Five groups, each consisting of 5 individuals, of 4-week-old male Wistar rats (Rattus novergicus) were used. Rats were treated with brown rice pellet, with a combination of herbal extracts for 3 weeks. Measurement of rat condition were conducted from the beginning until day 22 of treatment, namely blood glucose level, weight gain and feed intake amount. Physiological profile at community level of intestinal microbiota from cecum was measured using BIOLOG Ecoplate ${ }^{\mathrm{TM}}$. During treatment, rats were kept in the SITH ITB Laboratory at $25^{\circ} \mathrm{C}$ with 12 hours of light and 12 hours of dark and handled according to the ITB Ethics Committee protocols. Feeding/intake groups were designed as shown in Figure 1, with baseline before treatment (induction) are not shown (different batch).

\subsection{Blood Glucose Level Test}

Blood glucose level was measured with ACCU-Chek Glucometer (Roche), before and after brown rice-herbal fed treatment, according to manufacturer instruction. Blood were drawn from orbital sinus using capillary tubes (80 IU/ml), where a drop of blood was then applied to the glucometer to read.

\subsection{Community-Level Physiological Profiling (CLPP)}

Each well of the BIOLOG ${ }^{\mathrm{TM}}$ plate was inoculated with $150 \mu \mathrm{L}$ of rat cecum sample and incubated at 20 to $30^{\circ} \mathrm{C}$. Changes of absorbance are measured over incubation period of $24 \mathrm{~h}$ at $590-600 \mathrm{~nm}$ wavelength. To obtain uniform suspension, homogenization procedure was done prior to each measurement. 


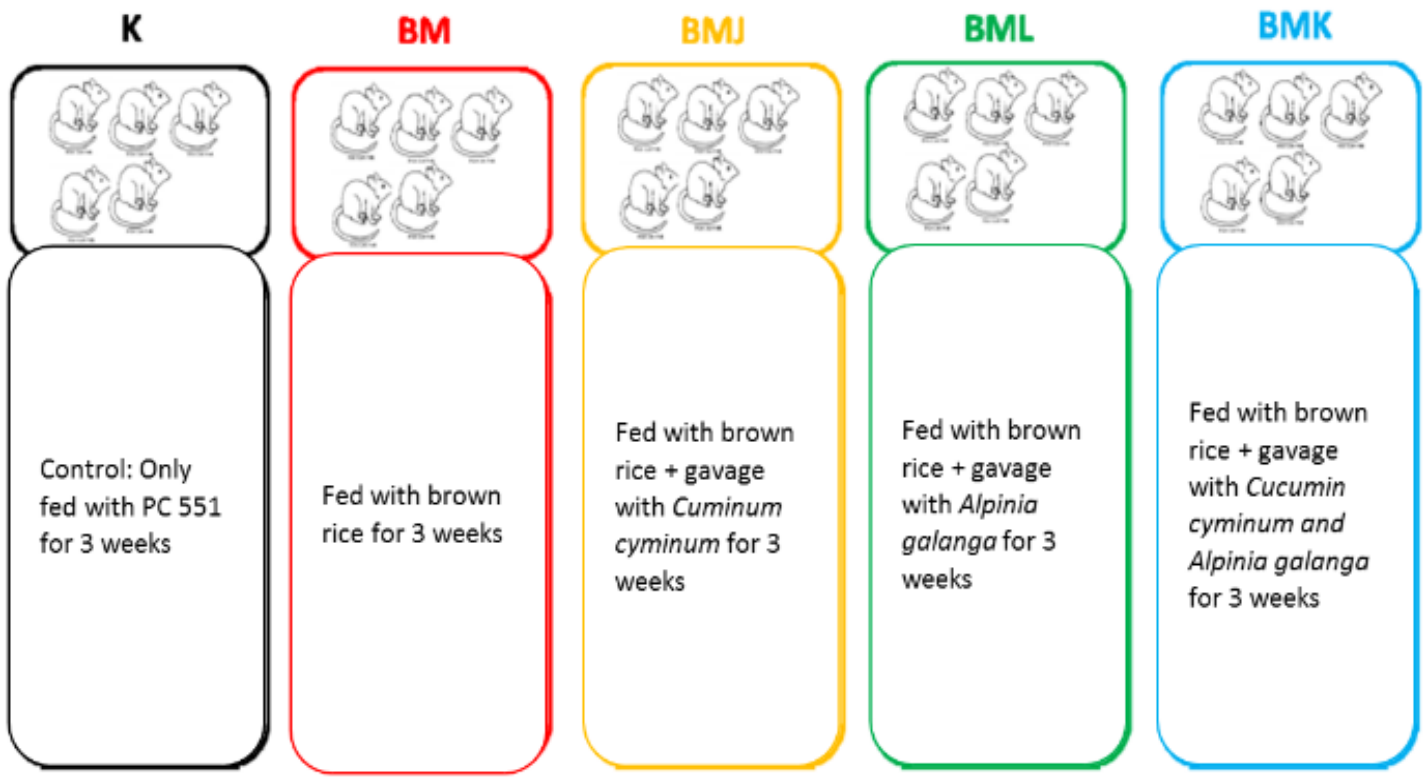

Figure 1. Research design and feeding scheme. Five groups of rats were established with $\mathrm{K}$ as control; BM is group fed with brown rice; BMJ is group fed with brown rice and C. cyminum extract; BML is group fed with brown rice and A. galanga extract; BMK is group fed with brown rice and extract of both C. cyminum and A. galanga

\subsection{Next Generation Sequencing (NGS) Analysis}

Cecum samples were analyzed through service provided by collaboration of Genetika Science (Indonesia) and Novagene (Singapore). DNA were isolated using Zymobiomics DNA Minikit following the manufacturer procedure. DNA samples were subjected to $16 \mathrm{~S}$ rRNA gene amplicon sequencing on Illumina Hiseq 2500 .

\subsection{Statistical Test}

Statistical tests were conducted using SPSS Statistics and collaborate with statisticians from the Statistical
Expertise Group of Faculty of Mathematics and Natural Sciences, ITB.

\section{Results and Discussion}

\subsection{Average Body Weight and Feed Consumption}

Experimental rats were handled according to the regulation and its condition were measured. The average body weight of rats before being treated was $260.43 \pm 12.29 \mathrm{~g}$ with a range of from 243.30 to $290.20 \mathrm{~g}$. Treatment with brown rice and herbal extracts were found to increase the rat body weight, similar to that of control (Figure 2).

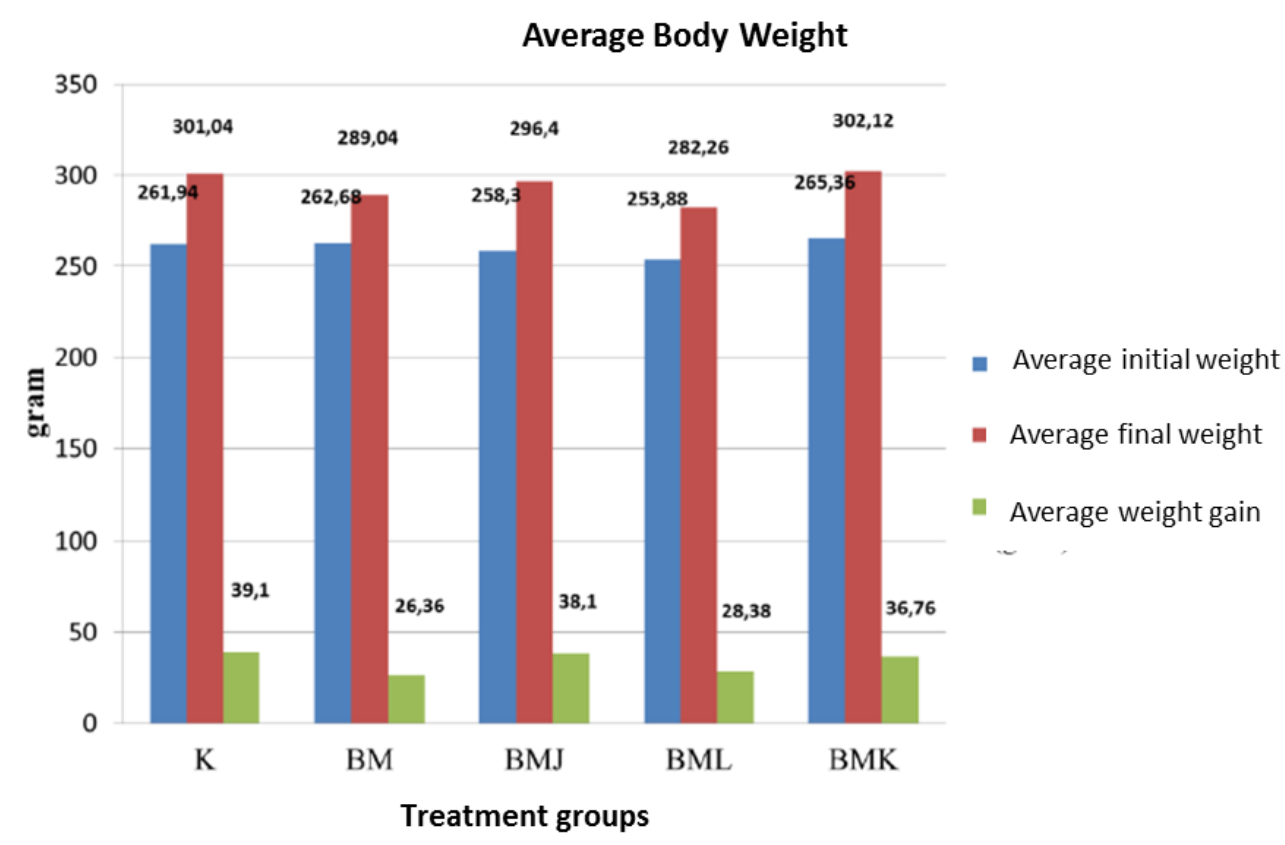

Figure 2. Average body weight of different groups of rats. Blue bar indicates the average initial weight (day 0), red bar indicates average final weight (day 21), and green bar indicates the average weight gain. Treatment groups coding are indicated as previously described. 
Table 1. Average feed consumption per day

\begin{tabular}{|c|c|c|c|c|c|}
\hline \multicolumn{7}{|c|}{ Treatment Groups } \\
\hline \multirow{2}{*}{$\begin{array}{c}\text { Average Feed } \\
\text { Weight (g) }\end{array}$} & K & BM & BMJ & BML & BMK \\
\cline { 2 - 6 } & $28.48 \pm 5.33$ & $16.80 \pm 3.23$ & $19.13 \pm 3.04$ & $19.00 \pm 3.79$ & $19.35 \pm 3.08$ \\
\hline
\end{tabular}

As shown in Figure 2, rats in all treatment groups experienced increase in body weight with the highest average increase in the control group $(39.1 \pm 19.47 \mathrm{~g})$, while the lowest average weight gain was found in those fed with only brown rice $(\mathrm{BM})(26.36 \pm 7.73 \mathrm{~g})$. In addition to the average body weight, we also measured the average amount of feed consumed by rats in all treatment groups in 21 days, as shown in Table 1.

Rats in the control treatment group $(\mathrm{K})$ were known to have the highest average feed consumption compared to the other treatment groups, while the treatment group which was only given the brown rice mixture had the lowest average feed consumption. Accordingly, this is directly proportional to the average weight gain of rats in Figure 2. Furthermore, the treatment was not detrimental for the rats.

\subsection{Blood Glucose Level}

Blood glucose levels in all treatment groups were measured on day 0 (initial day) and day 22 (after final day). As shown in Figure 3, almost all average blood glucose levels were decreased except in the BML treatment group which was fed with a mixture of brown rice and Alpinia galanga extract exhibiting slight increase. However, rats blood glucose levels before and after treatment remained at normal limits $(<200 \mathrm{mg} / \mathrm{dL})$. In general, brown rice, $A$. galanga extract, and $C$. cyminum extract treatment have blood glucose lowering effect.

\subsection{Gut Microbiota Activity}

Physiological profiling at community level was carried out to provide information regarding the activity of the microbiota contained within rat intestines. The CLPP test results showed that the treatment group which was given brown rice and herbal extracts (A. galanga and C. cyminum) had the greatest microbiota activity as indicated by the average well color development (AWCD) index (Figure 4).

AWCD index indicates that the combination of brown rice and herbal extracts (A. galanga and $C$. cyminum) provides an environment that promote stable increase of microbiota population. The index can also distinguish microbiota based on their activity in degrading certain substrates contained in EcoPlate. These substrates are further grouped into carbohydrates, polymers, proteins, phenols and carboxylic acids. Specific substrate type utilization for each experimental group are shown Figure 5. It is exhibited that the group provided with brown rice and both herbal extracts (BMK) has the highest degradation activity in almost all type of substrates. This activity increased as time progressed.

The elevated degradation activity of various types of substrate is related to the microbial diversity level of gut microbiota. As availability of diverse substrates, including that of fiber, polyphenol, polysaccharides and variety of other compounds promote the growth of various types of microbes. In turn, these gut microbiotas would generate products that both directly and indirectly implicate the health of the host [17].

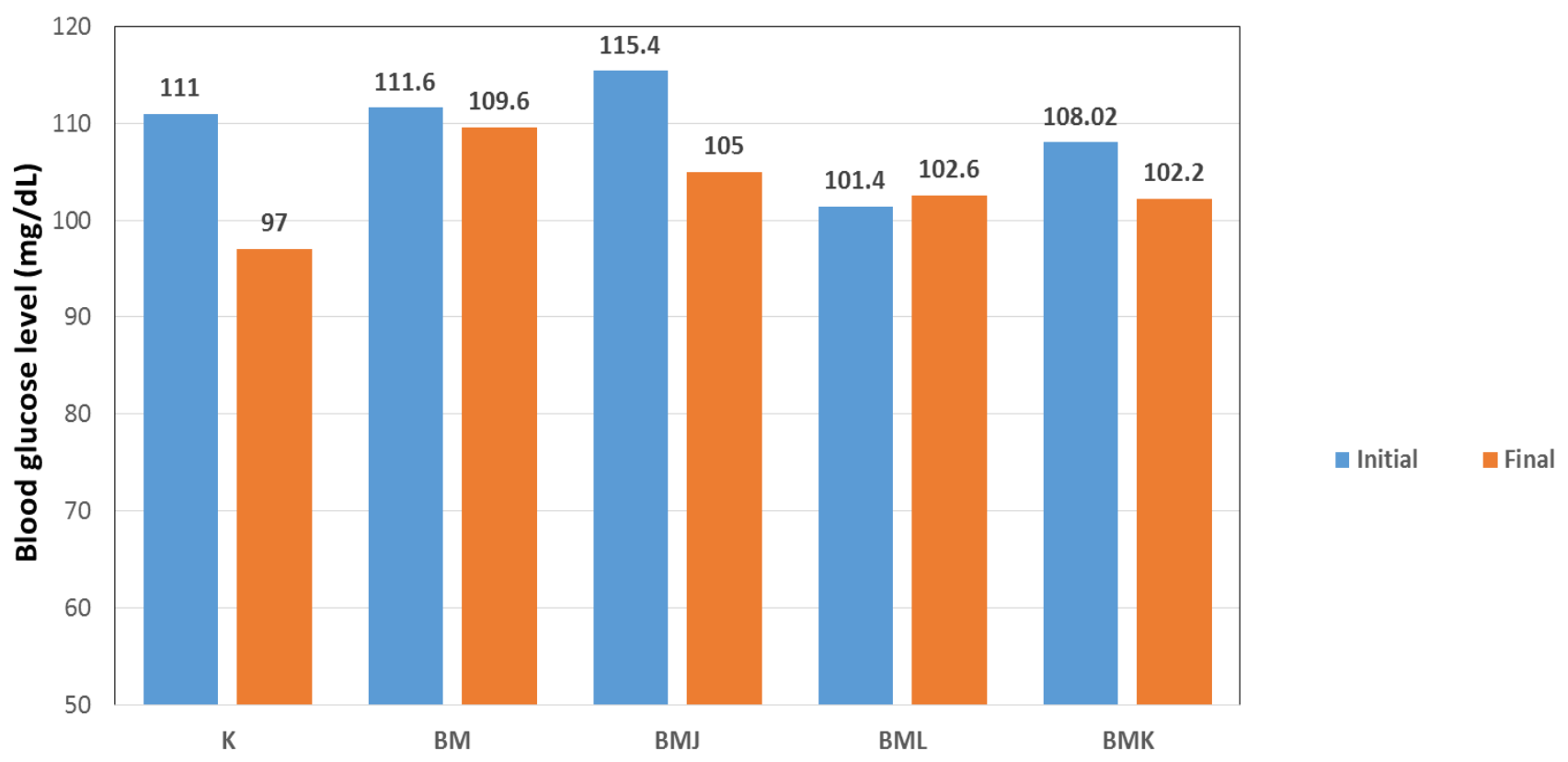

Treatment groups

Figure 3. Average blood glucose level of rat in all treatment groups. Blue bar indicates the average of initial level of blood glucose, whereas orange bar indicates the average of final level of blood glucose in each of the groups. 


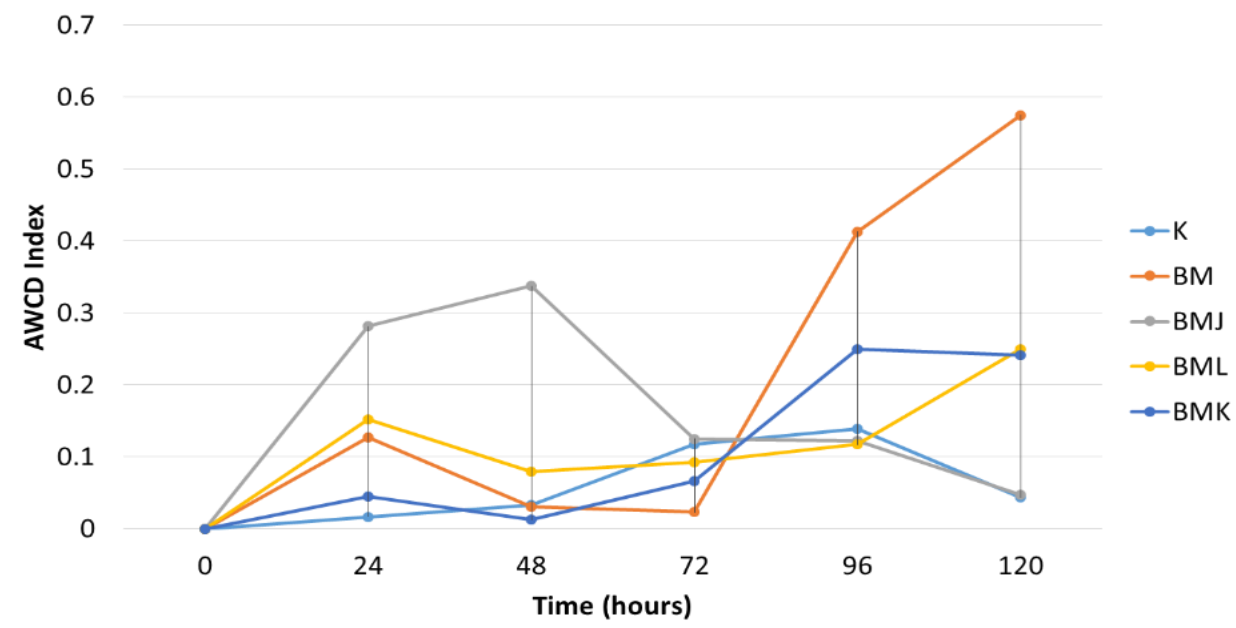

Figure 4. Average Well Color Development (AWCD) in CLPP measurement

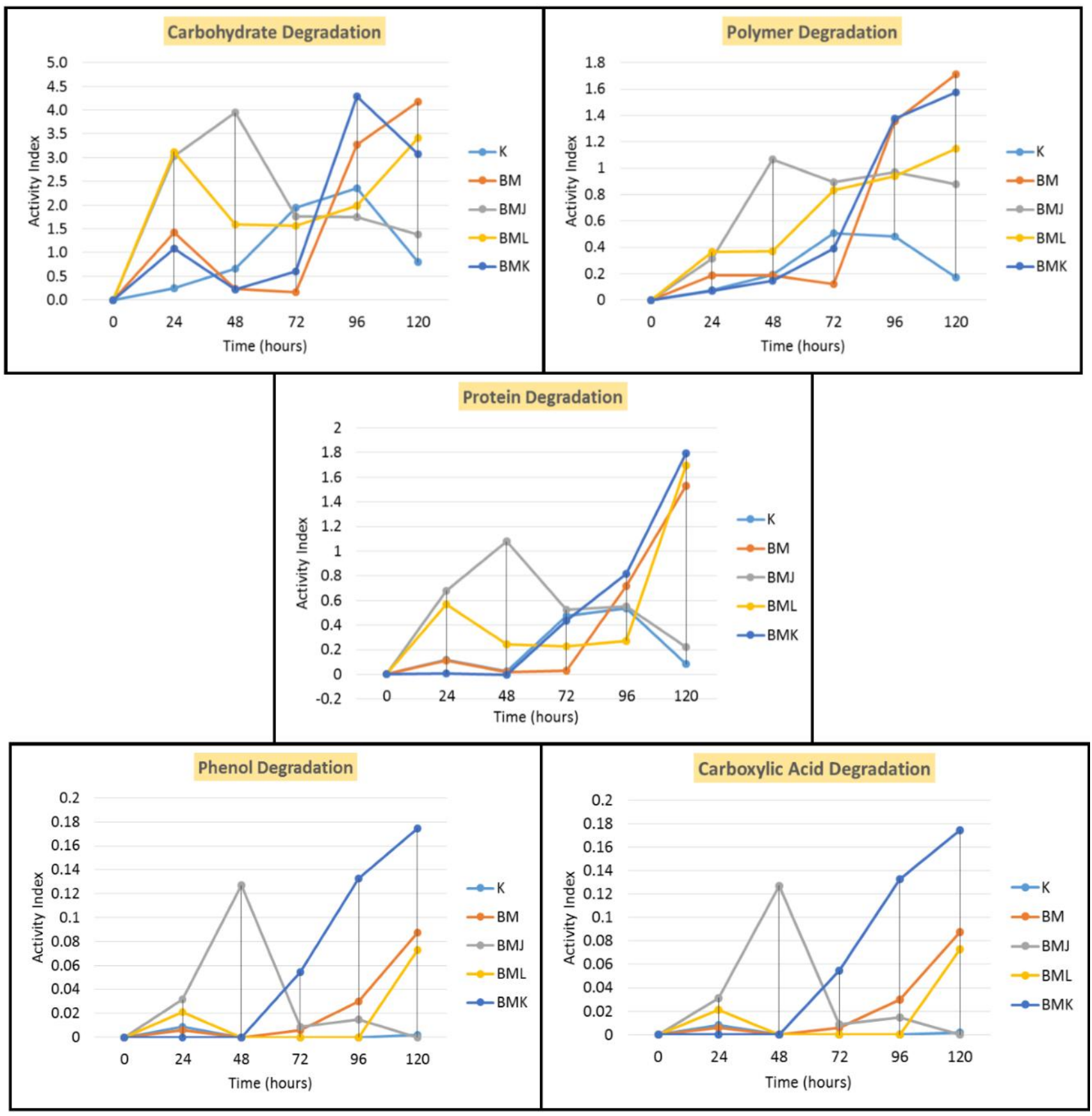

Figure 5. Average substrate degradation activity by gut microbiota in rat intestine 
Elevated carbohydrate degradation activity indicates high population of carbohydrate degrading bacteria in the gut, such as Bacteroides spp. [18,19]. In addition, this type of bacteria usually also possesses protein degrading activity, along with Propionibacteria[20]. We also suspected the occurrence of Clostridium and Eubacterium due to high phenol degradation activity in the mice gut. As phenol groups are known as an anti-bacterial component that could suppress pathogenic population in the intestine, interaction between gut microbiota and phenol degradation could improve host health status and prevent intestinal diseases [21].

Previous studies have shown that brown rice is constituted of non-digestible carbohydrate, such as non-starch polysaccharides, resistant starches, and fructo-oligosaccharides, that largely uprocessed in the small intestine and fermented in the large intestine. These types of carbohydrates that are hard to digest can modulate the gut microbiota [22].

Alpinia and Cuminum are also known have the ability to modulate the gut microbiota. Dua et al. stated that Cuminum has an antimicrobial potential that work against the gastroenteritis-causing bacteria such as E. coli and $P$. aeruginosa, and $B$. pumilus which can cause abdominal cramps [23]. While Xie, et al., stated that the extract of Alpinia can increase the population ratio of Bacteroides and Firmicutes, the most abundance bacteria in the gut. In addition, providing Alpinia extract to T2DM mice (Type 2 Diabetes Mellitus) can reduce blood glucose levels and significantly reduce damage of renal pathology by modulating the gut microbiota composition [24].

\subsection{NGS Analysis of Gut Microbiota}

By reducing multi-dimensional data, we determined the principal coordinates based on distances between dietary treatment and visualized differences among groups of diets. As observed in Figure 6, all types of diets are scattered with the exception of those supplementated with C. cyminum, either with or without addition of A. galanga. The result indicates that supplementation with brown rice already alters the microbiome composition, whereas addition of individual plant extracts would intensify the difference of nutritional constitutes generating different microbial community composition. However, complementation of both plant extracts induces microbial community composition that is different from that of only single plant extracts and seems to be closer to that of addition with $C$. cyminum. Although need further confirmation, this could indicate that chemical constitution of the $C$. cyminum have greater effect in promoting specific microbial community.

In terms of taxonomic alpha diversity, there was significant difference between that of control standard feed with all of the other diets supplemented with brown rice. Control feed significantly promoted lesser number of Operational Taxonomic Units (OTUs) observed, species richness, and diversity (Figure 7). Supplementation of brown rice significantly promoted the increase of species richness/diversity and the evenness of community. Addition of plant extracts, however, decreased the total number of OTUs indicating that the presence of specific plant compounds provided growth constraints for specific OTUs. Relationship between dietary types based on their unique OTUs and shared OTUs are illustrated in Figure 8. Based on the abundance of phyla (Figure 9), different diet types promote growth of different phyla. For example, the standard feed promote growth that of Lentisphaerae, whereas supplementation of two plant extracts promotes Synergistetes which is found very low in other dietary types.

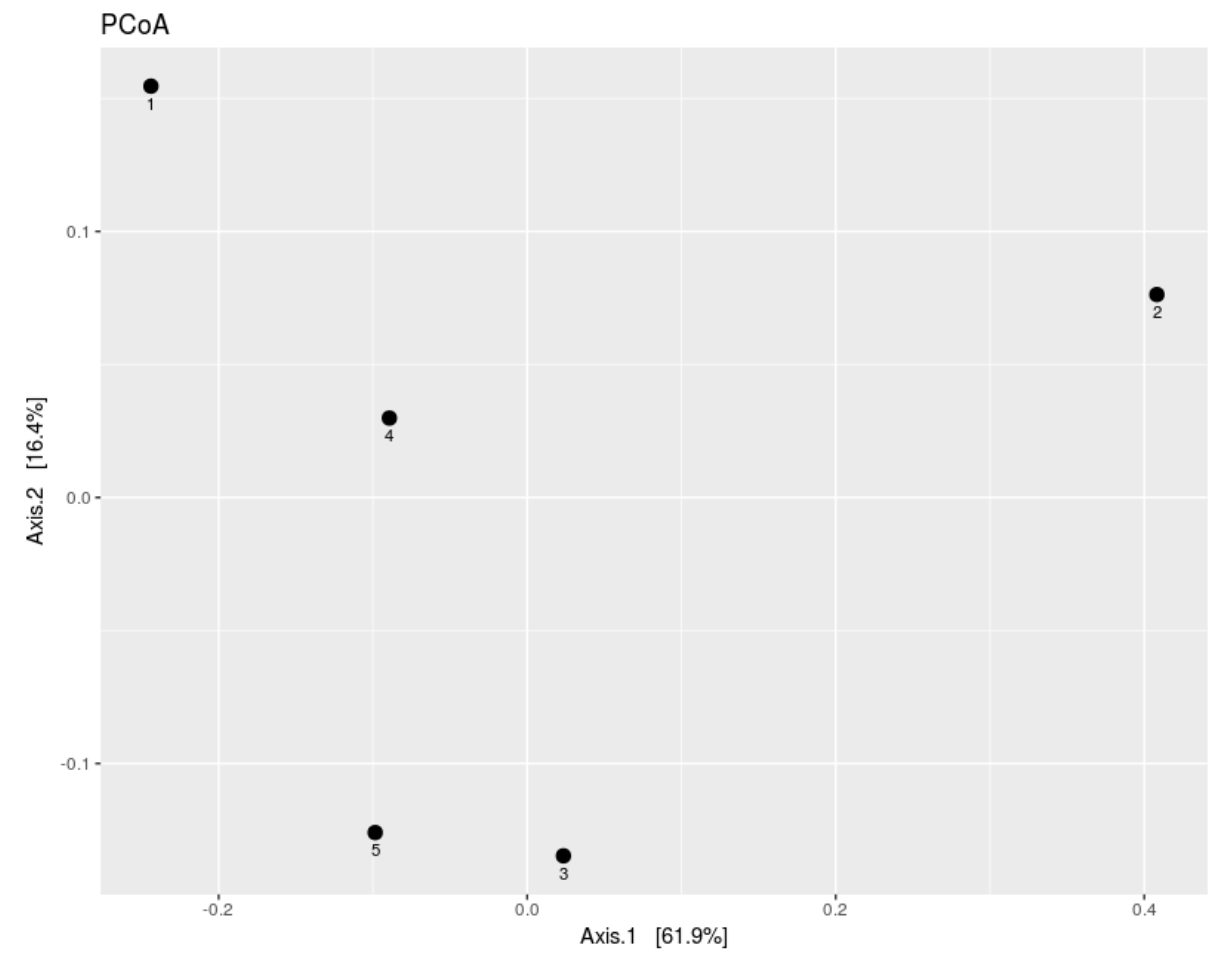

Figure 6. Principle Coordinates Analysis plot. Principle Coordinates Analysis (PCoA) based on dissimilarities of microbial communities found in the five different diets (numbered as control standard feed [1]; supplemented with brown rice [2]; supplemented with brown rice + Cuminum cyminum [3]; supplemented with brown rice + Alpinia galanga [4]; and supplemented with brown rice + C. cyminum + A. galangal [5] 


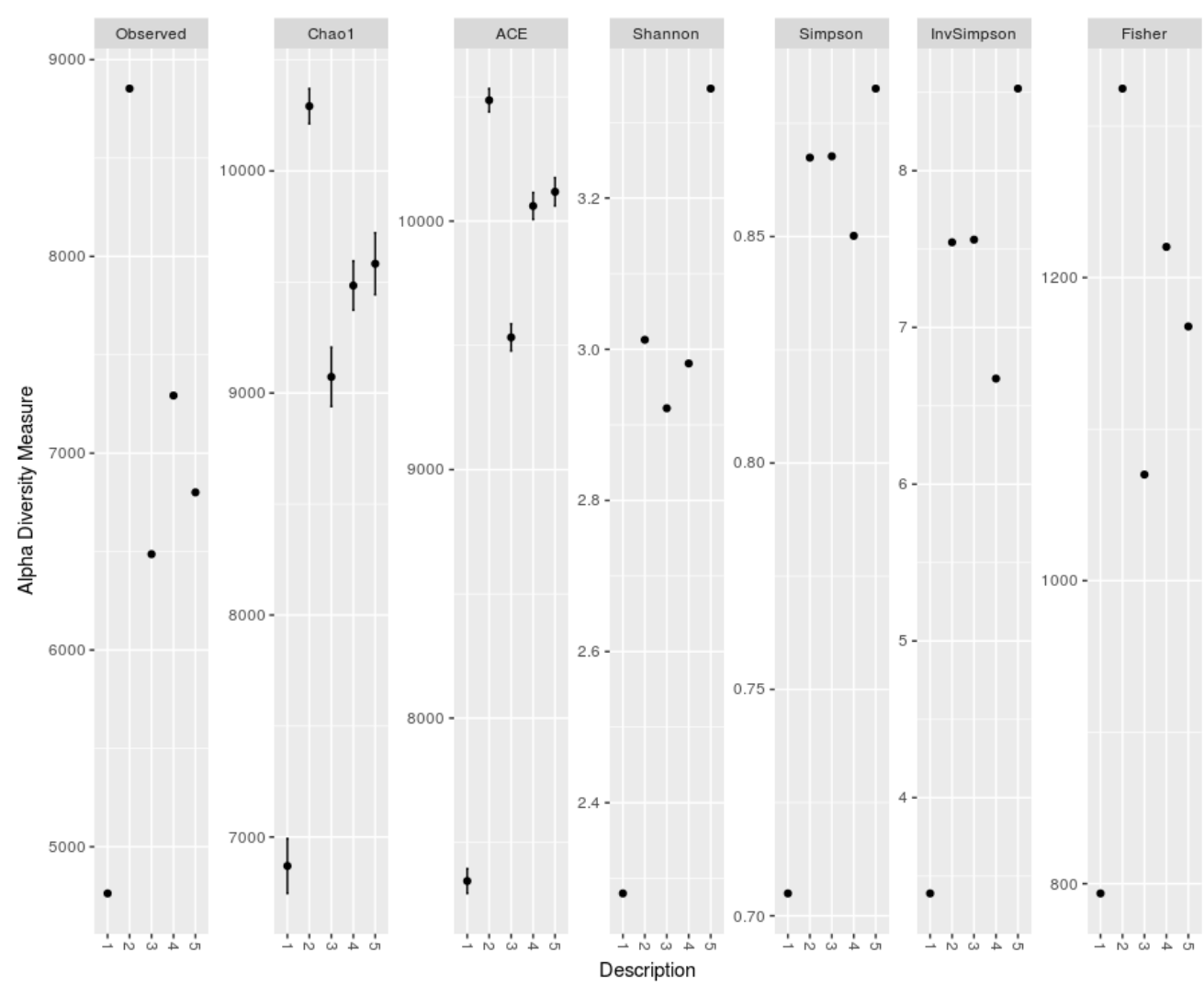

Figure 7. Alpha diversity measures for five diet types. Control diets constituting only standard feeds [1], significantly differs from all other type of diets (supplemented with brown rice [2]; brown rice + Cuminum cyminum [3]; brown rice + Alpinia galanga [4]; and brown rice + C. cyminum + A. galanga [5]) according to all of diversity measures

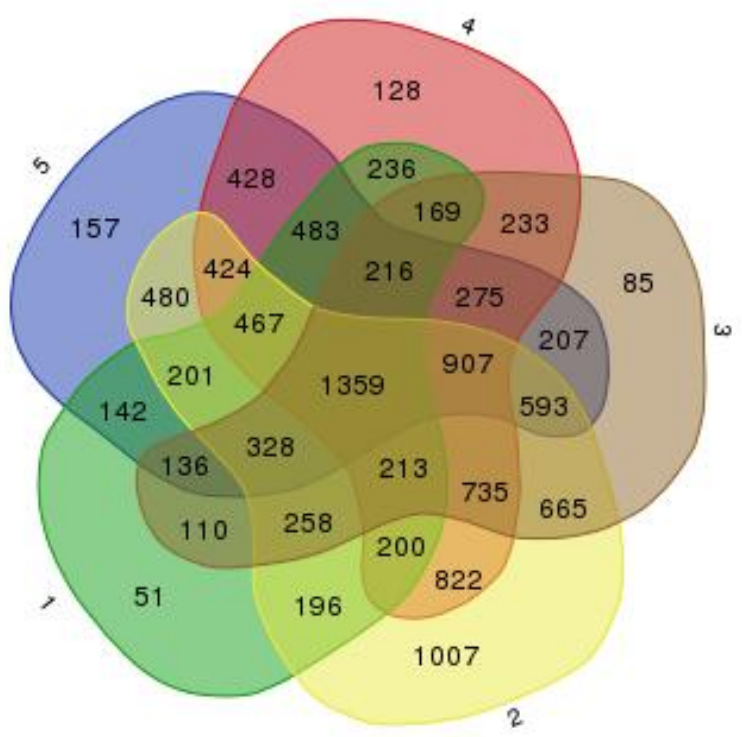

Figure 8. Venn diagram of OTUs per sample. This illustrate the relationship between and among sets, groups of samples with their own unique OTUs and those that share OTUs in common. Number on the outside of the Venn diagram indicate different dietary supplements as follow: standard feed [1]; supplemented with brown rice [2]; brown rice + Cuminum cyminum [3]; brown rice + Alpinia galanga [4]; and brown rice $+C$. cyminum + A. galanga [5]

Phenolic compounds, including that from A. galanga and C. cyminum extracts, are known to influence the relative abundance of different bacterial groups within gut microbiota. It reduces the numbers of potential pathogens, including Spirochaetes and Lentisphaerae, and promote the growth of many beneficial bifidobacterial and lactobacilli. It is shown that providing combination of brown rice and both herbal extracts, promote the most abundant beneficial microbes within the rat gut microbiota.

These results suggest that the combination of brown rice with bioactive compounds contained in A. galanga and $C$. cyminum herbal extracts provides good conditions for microbiota in rat intestines. Generally, the high diversity of gut microbiota is considered as one indicator of healthy gut [17]. 


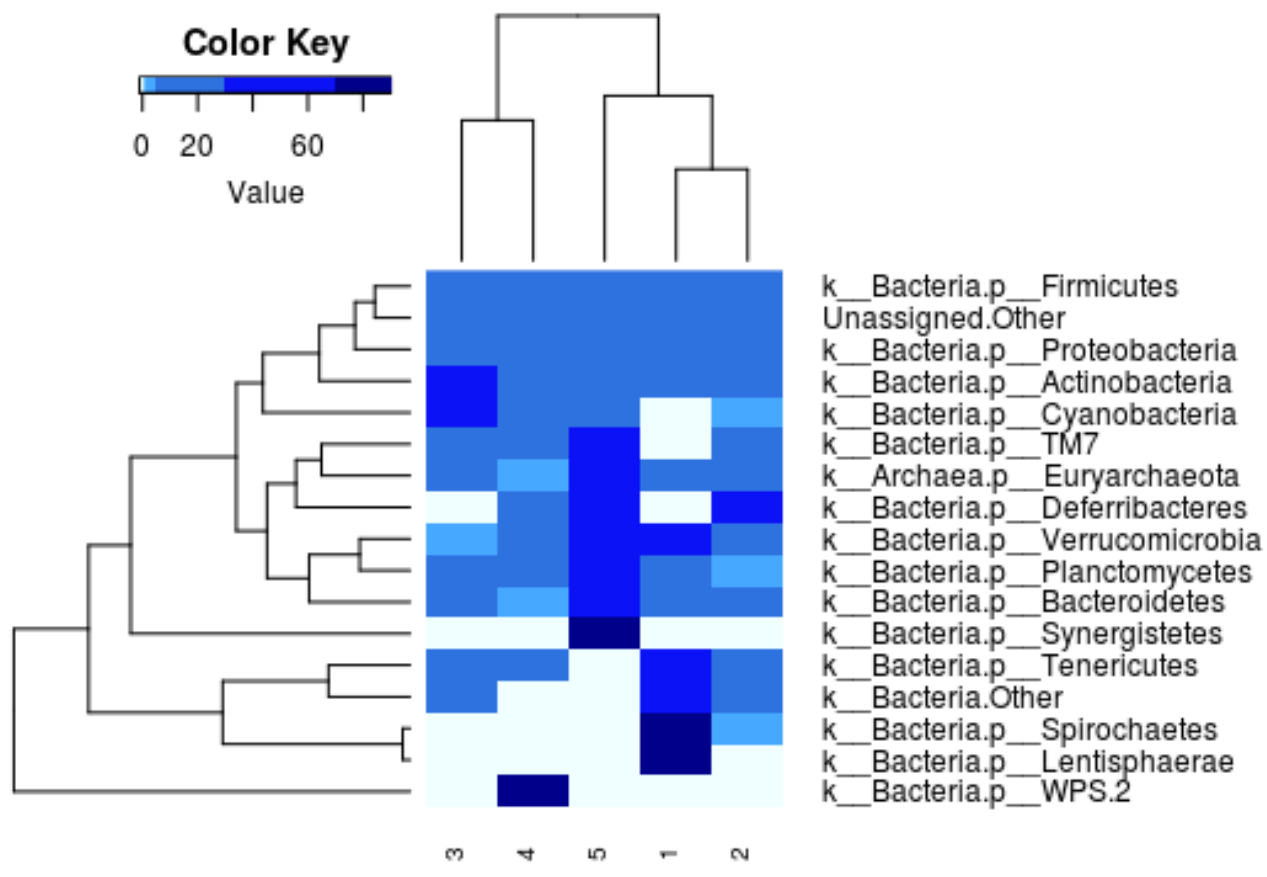

Figure 9. Heatmap of phyla in different dietary supplements. Relative abundances of phyla is illustrated using different shades of color for each type of diets. The higher the values, the darker the represented color. Phylogeny relatedness of phyla is shown in the top clustering, whereas similarity between samples are on left clustering. Hierarchical clustering of samples was done with pearson correlation and average linkage algorithm. Dietary supplements are as follow: standard feed (1); supplemented with brown rice (2); brown rice + Cuminum cyminum (3); brown rice + Alpinia galanga (4); and brown rice + C. cyminum + A. galanga $(5)$.

\section{Conclusion}

In conclusion, our results have shown that brown rice, A. galanga extract, C. cyminum extract, and their combination have the effect of lowering blood glucose level and increase gut microbial activity. Furthermore, supplementation with brown rice already alters the microbiome composition. Therefore, these findings suggest that brown rice diet and herbal (A. galanga and C. cyminum) extract administration are good for health by altering metabolism.

\section{References}

[1] Vijay-Kumar, M., Aitken, J.D., Carvalho, F.A., Cullender, T.C., Mwangi, S., Srinivasan, S., Sitaraman, S.V., Knight, R., Ley, R.E., Gewirtz, A.T., "Metabolic syndrome and altered gut microbiota in mice lacking Toll-like receptor 5", Science, 328 (5975), 228-231, 2010.

[2] Cornier, M.A., Dabelea, D., Hernandez, T.L., Lindstrom, R.C., Steig, A.J., Stob, N.R., Van Pelt, R.E., Wang, H., and Eckel, R.H., "The metabolic syndrome". Endocrine reviews, 29 (7), 777-822, 2008.

[3] Louis, P., Scott, K.P., Duncan, S.H., and Flint, H.J., "Understanding the effects of diet on bacterial metabolism in the large intestine", Journal of Applied Microbiology, 102 (5), 1197-1208, 2007.

[4] Xu, J., Lian, F., Zhao, L., Zhao, Y., Chen, X., Zhang, X., Guo, Y., Zhang, C., Zhou, Q., Xue, Z., Pang, X., Zhao, L. and Tong, X., "Structural modulation of gut microbiota during all eviation of type 2 diabetes with a Chinese herbal formula", The ISME Journal, 9, 552-562, 2015.

[5] Martinez, I., Lattimer, J.M., Hubach, K.L., Case, J.A., Yang, J., Weber, C.G., Louk, J.A., Rose, D.J., Kyureghian, G., Peterson, D.A., Haub, M.D., and Walter, J., "Gut microbiome composition is linked to whole grain-induced immunological improvements", The ISME Journal, 7 (2), 269-280, 2013.
[6] Elfahmi, Woerdenbag, H.J., and Kayser, O., "Jamu: Indonesian traditional herbal medicine towards rational phytopharmacological use", J. Herbal Med., 2014.

[7] World Health Organization: Population-based approaches to childhood obesity prevention. 2012.

[8] Lee, S.J., Bose, S., Seo, J.G., Chung, W.S., Lim, C.Y., and Kim, H., "The effects of co-administration of probiotics with herbal medicine on obesity, metabolic endotoxemia and dysbiosis: Arandomized double-blind controlled clinical trial", Clinical Nutrition, 33, 2014.

[9] Nguyen, T.L., Vieira-Silva, S., Liston, A., and Raes, J., "How informative is the mouse for human gut microbiota research?", Disease Models and Mechanisms, 8 (1), 1-16, 2015.

[10] Rastelli, M., Knauf, C., and Cani, P.D., "Gut Microbes and Health: A Focus on the Mechanisms Linking Microbes, Obesity, and Related Disorders", Obesity, 26, 792-800, 2018.

[11] Rajoka, M.S.R., Shi, J., Mehwish, H.M., Zhu, J., Li, Q., Shao, D., Huang, Q., and Yang, H., "Interaction between diet composition and gut microbiota and its impact on gastrointestinal tract health", Food Science and Huamn Wellness, 6(3), 121-130, 2017.

[12] Lyu, M., Wang, Y., Fan, G., Wang, X., Xu, S., and Zhu, Y., "Balancing Herbal Medicine and Functional Food for Prevention and Treatment of Cardiometabolic Diseases through Modulating Gut Microbiota", Frontier in Microbiology, 8, 2146, 2017.

[13] Kim, H.B., and Isaacson, R., "The pig gut microbial diversity: understanding the pig gut microbial ecology through the next generation high throughput sequencing", Veterinary Microbiology, 177 (3-4), 242-251, 2015.

[14] Mayo, B., Rachid, C.T.C.C., Alegria, A., Leite, A.M.O., Peixoto, R.S., and Delgado, S., "Impact of Next Generation Sequencing Techniques in Food Microbiology", Current Genomics, 15, 293-309.

[15] Dhandapani, S., Subramanian, V.R., Rajagopal, S., and Namasivayam, N., "Hypolipidemic Effect of Cuminum Cyminum on Alloxan-Induced Diabetic Rats", Pharmacological Research, 46 (3), 251-255, 2002.

[16] Verma, R.K., Mishra, G., Singh, P., Jha, K.K., and Khosa, R.L., "Anti-diabetic Activity of Methanolic Extract of Alpinia galanga Linn. Aerial Parts in Streptozotocin Induced Diabetic Rats", Ayu, $36(1), 91-95,2015$.

[17] Valdes, A.M., Walter, J., Segal, E., and Spector, T.D., "Role of the gut microbiota in nutrition and health". BMJ, 361, 2018. 
[18] Flint, H.J., Scott, K.P., Dunca, S.H., Louis, P., and Forano, E., "Microbial degradation of complex carbohydrates in the gut", Gut Microbes, 3 (4), 289-306, 2012.

[19] Cartmell, A., Lowe, E.C., Basle, A., Firbank, S.J., Ndeh, D.A., Murray, H., Terrapon, N., Lombard, V., Henrissat, B., Turnbull, J.E., Czjzek, M., Gilbert, H.J., and Bolam, D.N., "How members of human gut microbiota overcome the sulfation problem posed by glycosaminoglycans", PNAS, 114 (7), 7037-7042, 2017.

[20] Macfarlane, G.T., Cummings, J.H., and Allison, C., "Protein Degradation by Human Intestinal Bacteria", Journal of General Microbiology, 132, 1647-1656, 1986.

[21] Selma, M., Espin, J.C., and Barberan, F.A.T., "Interaction between Phenolics and Gut Microbiota: Role in Human Health",
Journal of Agriculturaland Food Chemistry, 57, 6485-6501, 2009.

[22] Han, F., Wang, Y., Han, Y., Zhao, J., Han, F., Song, G., Jiang, P., and Miao, H., "Effect of whole-grain rice and wheat on composition of gut microbiota and short-chain fatty acids in rats", Journal of Agricultural and Food Chemistry, 2018.

[23] Dua, A., Gaurav, G., Balkar, S., and Mahajan, R., "Antimicrobial Properties of Methanolic Extract of Cumin Cuminum Cyminum Seeds", IJRAP, 4(1), 104-107, 2013.

[24] Xie, Y., Xiao, M., Ni, Y., Jiang, S., Feng, G., Shenggang, S., and Du, G., "Alpinia oxyphylla Miq. Extract Prevents Diabetes in Mice by Modulating Gut Microbiota", Journal of Diabetes Research, 2018.

(C) The Author(s) 2019. This article is an open access article distributed under the terms and conditions of the Creative Commons Attribution (CC BY) license (http://creativecommons.org/licenses/by/4.0/). 